\title{
Separation of Line Drawings Based on Split Faces for 3D Object Reconstruction
}

\author{
Changqing Zou ${ }^{1,2,3}$, Heng Yang ${ }^{4}$, Jianzhuang Liu ${ }^{1,5,6}$ \\ ${ }^{1}$ Shenzhen Key Lab for CVPR, Shenzhen Institutes of Advanced Technology, China \\ ${ }^{2}$ Department of Physics and Electronic Information Science, Hengyang Normal University, Hengyang, China \\ ${ }^{3}$ University of Chinese Academy of Sciences, Beijing, China \\ ${ }^{4}$ Queen Mary University of London, London, UK \\ ${ }^{5}$ Department of Information Engineering, The Chinese University of Hong Kong, Hong Kong \\ ${ }^{6}$ Media Lab, Huawei Technologies Co. Ltd., China \\ aaronzou1125@gmail.com, heng.yang@qmul.ac.uk, liu.jianzhuang@huawei.com
}

\begin{abstract}
Reconstructing $3 D$ objects from single line drawings is often desirable in computer vision and graphics applications. If the line drawing of a complex $3 D$ object is decomposed into primitives of simple shape, the object can be easily reconstructed. We propose an effective method to conduct the line drawing separation and turn a complex line drawing into parametric $3 D$ models. This is achieved by recursively separating the line drawing using two type$s$ of split faces. Our experiments show that the proposed separation method can generate more basic and simple line drawings, and its combination with the example-based reconstruction can robustly recover wider range of complex parametric $3 D$ objects than previous methods.
\end{abstract}

\section{Introduction}

Reconstructing 3D objects from single line drawings is one of the important research topics in computer vision. The related applications of 3D reconstruction from line drawings include: providing flexible sketching interfaces for conceptual designers who prefer the pencil and paper over the mouse and keyword in current CAD systems $[3,4,9]$ (the pipeline of a typical sketch-based modeling system is shown in Fig. 1), interactively generating 3D models from images [5, 12, 19, 22], automatically converting existing industrial wireframe models to solid models [2,3], and providing user-friendly query input interface for $3 \mathrm{D}$ object retrieval from large 3D object databases and the Internet $[14,16]$.

Many methods have been proposed for automatically reconstructing 3D objects from single line drawings $[5,7,9,10,11,15,17,19,20]$. Among these methods, the two in [11] and [20] can handle more complex object$\mathrm{s}$ than the others. In [11], the authors propose to separate a complex line drawing into simpler line drawings by the internal faces within the line drawing, then independently reconstruct the 3D shapes from these simpler ones using an optimization-based algorithm, finally obtaining a complete object by merging these 3D shapes together. The authors in [20] recover parametric 3D models from line drawings using the similar steps as that of [11]. The method first decomposes a line drawing into simpler ones using the algorithm in [11], then matches each decomposed line drawing with several basic 3D models in a database, finally uses a graphical model based algorithm to derive the complete 3D model that fits the input line drawing best.

In general, both of the above two methods benefit from the line drawing decomposition based on internal faces. However, internal faces do not appear frequently in some line drawings. One example is given in Fig. 2(a) where there is only one internal face (Fig. 2(b)). Its separation is shown in Fig. 2(c). We can see that there still exist complex line drawings (partitions) after decomposition, which limits the application of the reconstruction methods. To solve the problem, Xue et al. [21] proposed an object cut based line drawing separation algorithm. Compared to the internal face method in [11] that only includes original edges in the input line drawing, an object cut may include newly generated edges (i.e., an internal face is a special case of an object cut), hence can decompose wider range of line

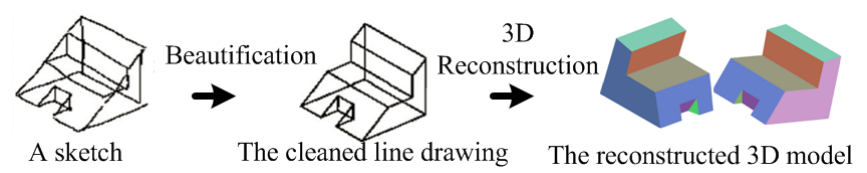

Fig. 1. A 3D modeling system based on sketching interfaces [5]. In this modeling system, 3D objects are reconstructed from line drawings, which are obtained by processing users' freehand sketches. 


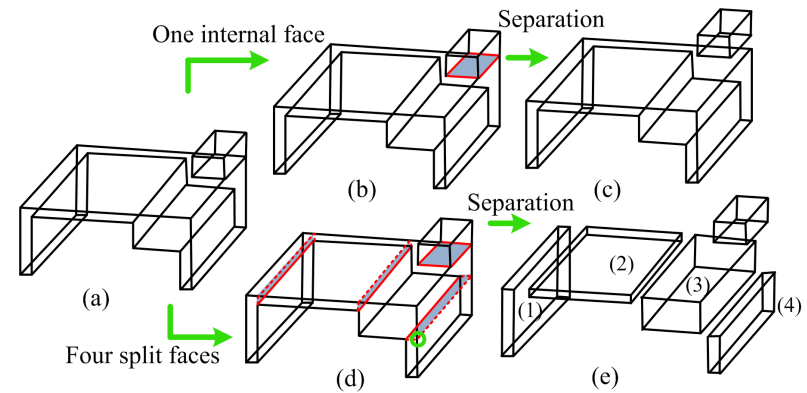

(d)

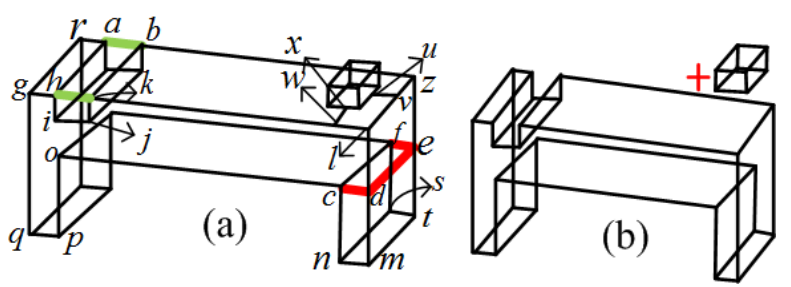

Fig. 3. Visualization of terms. In (a), cycle $(c, d, e, f, c)$ and $(h, k, b, a, h)$ are two split faces, $(g, q, p, o)$ is a chain consisting of three edges, Internal split edges $\{c, d\}$ and $\{d, e\}$ in red are of type $I$ and type $I I$, respectively. New edges $\{a, b\}$ is an external split edge. Vertices $d$ and $e$ are two split vertices. Face $(g, q, p, o, c, n, m, l, k, j, i, h, g)$ is a concave face. Vertex $m$ is a boundary vertex of the three planes on which face $(m, l, z, t, m)$, face $(m, n, s, t, m)$, and face $(g, q, p, o, c, n, m, l, k, j, i, h, g)$ respectively lie. Hence, $g$ is also a $3 \mathrm{D}$-convex vertex. Edges $\{u, v\}$ and $\{w, x\}$ are two artificial lines indicating that the two cycles connected by them are coplanar. (b) shows the resulted submanifolds after removing these artificial lines.

drawings. However, the object cut based separation algorithm still does not completely resolve the problem of the separation of a complex line drawing that represents a solid object, since it is conditioned on at least the following two situations: 1) both of the endpoints of a new edge should be the original vertices in the input line drawing, and 2) a new edge should be parallel (or near parallel) to an original edge. As shown in Fig. 2b, the partition representing a desk (i.e. the partial line drawing consisting of partitions (1)-(4) in Fig. 2e) cannot be further separated by this method since no object cut can be generated.

In this work, we propose a new approach, which leverages split faces (see the next section for its definition) to separate a complex line drawing. Different from the internal face of which the edges and vertices are all from the input line drawing, an edge or vertex in a split face can be either newly generated or original. In fact, both the internal face in [11] and the object cut in [21] are only two special cases of our proposed split face. Compared to [11] and [21], the proposed split face based method can obtain better decomposition results (i.e. it handles wider range of line drawings). One example is shown in Fig. 2(e), 5 simpler partitions which represent 5 regular objects are obtained by the proposed algorithm, while only two partitions are obtained by [11] or [21]. In addition, our proposed method outperforms both [11] and [21] in terms of efficiency in the experiments.

\section{Assumption, Terminology and Preprocess- ing}

Similar to [11], the paper focuses on the 3D reconstruction of manifolds. On the surface of a manifold, every point has a neighborhood topologically, that is equivalent to an open disk in the 2D Euclidean space [1]. A line drawing in this paper is assumed to be an orthogonal projection of the edges of a 3D planar-faced manifold in a generic view, with

hidden lines and vertices visible. For better understanding of the contents, here we give some terms that are used in the rest of the paper.

1. Cycle. A cycle is formed by a sequence of vertices $v_{0}, \ldots v_{n}$, where $n \geq 3, v_{0}=v_{n}$, the $n$ vertices are distinct, and there exists an edge connecting $v_{i}$ and $v_{i+1}$ for $i=0,1, \ldots, n-1$. A cycle is denoted by $\left(v_{0}, \ldots, v_{n}\right)$ [11] [13].

2. Face (real face). A face is a flat patch of a manifold bounded by a cycle. A face can be denoted by the same way as a cycle $[9,10,11]$.

3. Chain. A continuous part of a cycle is called a chain [9].

4. Artificial line. An artificial line is a line used to indicate the coplanarity of two cycles [2][13].

5. Degree of a vertex. The degree Degree $(v)$ of a vertex $v$ is the number of edges adjacent to $v$ [13].

6. Internal Face. An internal face is a face inside a manifold only with its edges visible on the surface. It is not a real face but is formed by gluing two manifolds together [11].

7. Cut-set. Let $P(G)=\left\{G_{0}, G_{1}, \ldots, G_{n}\right\}$ be a partition of the vertex set $V$ of a graph $G=(V, E)$, the cut-set of $P(G)$ is the set of edges with endpoints in different subsets of $P(G)$.

8. Vertex set of a face. The vertex set $V \operatorname{er}(f)$ of a face $f$ is the set of all the vertices of $f$.

9. Edge set of a face. The set of all the edges of a face $f$ is denoted by $\operatorname{Edge}(f)$.

10. Neighboring face. Two faces $f_{a}$ and $f_{b}$ are called two neighboring faces if $\operatorname{Edge}\left(f_{a}\right) \cap \operatorname{Edge}\left(f_{b}\right) \neq \emptyset$.

11. Split face. A split face is either (1) a planar cycle on the surface of a manifold $M$, formed by cutting $M$ with a plane, consisting of some edges of $M$ and/or 
new edges on the surface of $M$, with its enclosed region not on the surface of $M$, or (2) a planar cycle locating outside a manifold $M$, consisting of some edges of the manifold, into which another manifold can be formed by merging a part of $M$. A split face can also be classified into two types: 1) the ones without new edges, and 2) the ones with new edges. These two types of split faces are denoted by NS-Faces and $S$-Faces, respectively.

12. Internal split edge. An internal split edge is a new edge on the surface of a manifold, formed by cutting the manifold with a split face. A split edge can be of two types: 1) at most one of its endpoints is a new vertex, and 2) both of its endpoints are new vertices. These two types of split edges are called type $I$ and type II split edges, respectively.

13. External split edge. An internal split edge is a new edge outside the surface of a manifold, formed by merging a part of the manifold with a split face which locates outside the manifold.

14. Split vertex. The intersection of a split edge and an edge in a face $f$ is called an split vertex if $v \notin \operatorname{Ver}(f)$.

15. Concave face. A concave face is a face whose cycle is a concave polygon.

16. Boundary vertex of a plane. A vertex $v$ is called a boundary vertex of a plane $P$ if $v$ lies on the convex hull of all the vertices of the faces lying on $P$.

17. 3D-convex vertex. A vertex $v$ is called a $3 \mathrm{D}$-convex vertex if $v$ is a boundary vertex of each of the planes that intersect at $v$.

18. Extended line drawing. A line drawing added with new generated edges are called an extended line drawing.

In this work, we utilize the method in [13] to automatically obtain the faces in a line drawing before the line drawing decomposition. Artificial lines, added by the designer, are used to indicate the coplanarity of two cycles in solid modeling. Detecting artificial lines is an easy task based on the connection between an artificial line and the edges it connects to [11]. After removing the artificial lines in Fig. 3(a), the line drawing becomes two line drawings without artificial lines (Fig. 3(b)). We call the face identification, the detection and the removal of artificial lines preprocessing.

\section{Pipeline for line drawing Separation}

After preprocessing, a line drawing is separated into one or multiple line drawings (a line drawing remains undecomposed if it has no artificial lines). And then simper line drawings are obtained by executing such steps on each decomposed line drawing $G$ of the preprocessing stage:

1. identifying NS-Faces in $G$,
2. decomposing $G$ using the NS-Faces of Step 1,

3. generating S-Faces for each separated line drawing of Step 2,

4. decomposing each separated line drawing of Step 2 using the S-Faces of Step 3.

These four steps are detailed in the next two sections as follows: Step 1 and Step 2 in Section 4, Step 3 and Step 4 in Section 5.

\section{Decomposition by NS-Faces}

\subsection{Searching for NS-Faces}

A NS-Face in this work is equivalent to an internal face in [11], which is a face (not a real face) inside a manifold only with its edges visible on the surface. A NS-Face can also be regarded to be formed by gluing two faces belonging to two manifolds together. According to the topological structure of a line drawing, a NS-Face can be further classified into one of two types: 1) two cycles of the two glued faces have no contact, and 2) two cycles of the two glued faces have contact (partly or completely) [11]. In this paper, the NS-Faces of type 1, which use additional artificial lines to indicate the coplanarity of the two glued faces, have been removed in the preprocessing stage (i.e. the line drawing has been separated into multiple line drawings using the NS-Faces of type 1 in the preprocessing stage). Next, we discuss how to identify the NS-Faces of type 2 in a line drawing. For concision, "NS-Faces" is used to denote "NSFaces of type 2" in the remainder of this section.

Detecting NS-Faces in a line drawing is not a trivial problem. In this work, the searching for NS-Faces is preformed through a cycle searching scheme. To obtain an efficient searching, 6 properties related to NS-Face are used to eliminate the cycles that cannot be NS-Faces. Among these 6 properties, Properties 4-6 come from [11]. These three properties are developed based on the definition of an internal face, which also consist with that of a NS-Face in this work. Properties 1-3 are derived from the definition that a NS-Face is a planar cycle and some other properties of a manifold.

Proposition 1. A cycle of a NS-Face has at least one vertex of degree $n(n \geq 4)$.

Proposition 2. A cycle cannot be a NS-Face if the cycle has a 3 -convex vertex of degree 3.

Proposition 3. A cycle cannot be a NS-Face if the cycle shares two or more non-collinear edges with two neighboring faces.

The proofs of Proposition 1, 2, and 3 can be found in [23].

Proposition 4. A cycle cannot be a NS-Face if it is selfintersecting [11]. 


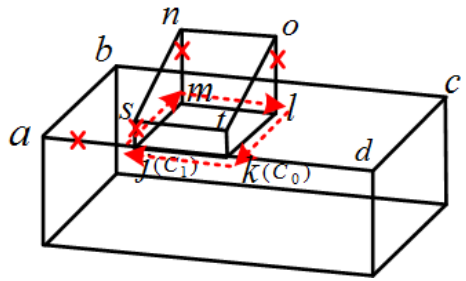

Fig. 4. Illustration of searching a NS-Face. The cycle $C$ of a NSFace is grown from edge $\{k, j\}$ with $k$ as the start vertex. The edges marked by $\times$ belong to the chains which cannot form a valid NS-Face, since these chains contradict one or more proposed propositions.

Proposition 5. A cycle cannot be a NS-Face if it has a chord inside it and the chord is on the surface of the manifold [11].

Proposition 6. A cycle cannot be a NS-Face if it has two non-collinear edges belonging to a face and there is an overlapping region between it and the face in the $2 D$ line drawing plane [11].

With these properties, we develop an algorithm to detect NS-Faces of a line drawing, which is summarized in Algorithm 1. It is a depth-first search algorithm with the properties incorporated to guide the search of valid NS-Faces. The properties can cut most fruitless branches during the search, and greatly speed up the algorithm. In the Algorith$\mathrm{m} 1$, an array $C$ is used to keep the vertices of a chain , and $C(0)$ and $C$ (last) denote the first and the last vertex, respectively. According to Proposition 1, the algorithm starts the search from the edges which contains a vertex of degree more than 3. Then a chain is grown to form the cycle of a NS-Face candidate under Proposition 2-5. Finally, Proposition 6 is used to remove invalid NS-Faces from the NS-Face candidates.

We take the line drawing in Fig. 4 to illustrate Algorithm 1. According to Proposition 1, two vertices $k$ and $j$ of edge $\{k, j\}$ are selected as the first and the second vertices to grow the cycle of a possible NS-Face. Then, the chain$\mathrm{s}$ passing through vertices $s$ and $a$ are omitted from the valid ones which can form NS-Faces according to Proposition 2, since $s$ and $a$ are both 3D-convex vertices. After this step, only one valid chain $(k, j, m)$ is obtained. Nex$\mathrm{t}$, the chain $(k, j, m)$ is grown up to $(k, j, m, l)$, since $n$ is also a 3D-convex vertex and $(k, j, m, n)$ is regarded as an invalid chain. Finally, we exclude the chain $(k, j, m, l, o)$ according to Proposition 2 or Proposition 3, and obtain the cycle of a valid NS-Face $(k, j, m, l, k)$, since it is compatible with Proposition 6 . The example demonstrates that the proposed propositions are very efficient to achieve a valid NS-Face.

\subsection{Decomposition based on NS-Faces}

Given a line drawing and the identified split faces and real faces (see Fig. 5(a) and (b)), an efficient algorithm, which is conducted on the dual graph of an input line drawing, is
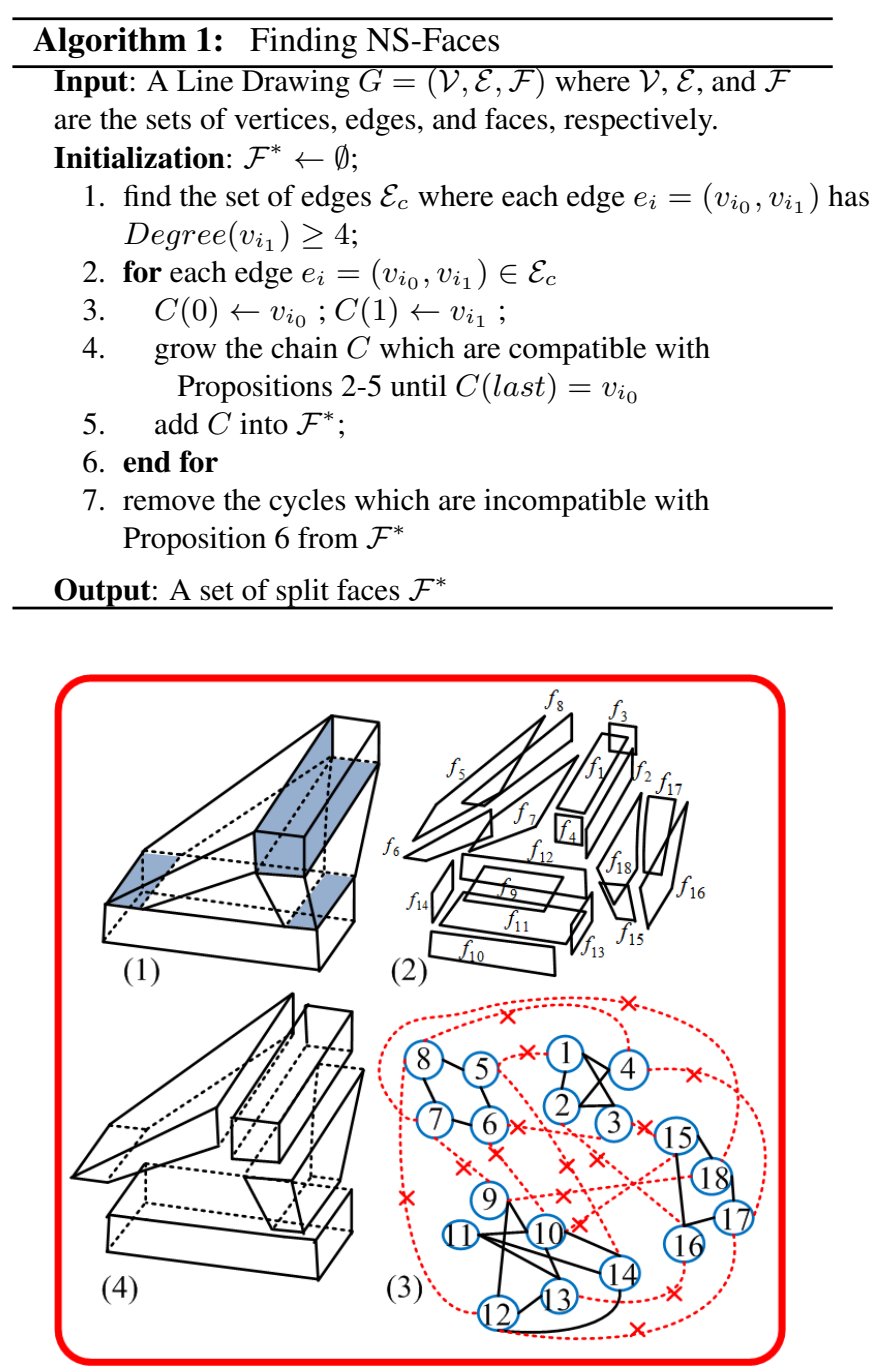

Fig. 5. An example of decomposing a line drawing using split faces. (1) The line drawing with four split faces shadowed. (2) 18 faces of the line drawing found in the preprocessing stage. (3) The built dual graph of the line drawing, with the edges of the split faces (marked by dotted lines) added into a cut-set. (4) The decomposed partitions with the split faces merged with their coplanar original faces.

used to decompose the line drawing into simpler partitions. Our decomposition algorithm is similar to the graph cut in graph theory [6], which separates a graph into two disjoint subsets. In this work, to facilitate the design of the decomposition algorithm, the dual graph $G$ of an input line drawing $G_{0}$ is firstly built, where each vertex denotes a face of $G_{0}$ and each edge of $G$ denotes the shared edge by two adjacent faces. Then all the edges of the split faces are mapped into a cut-set which is used to cut $G$ (see the example in Fig 5(c)), Finally isolated subsets, which correspond$\mathrm{s}$ to the decomposed partitions of the input line drawing, are obtained by removing the edges in the cut-set out of $G$. Algorithm 2 shows the steps of separating an input line 


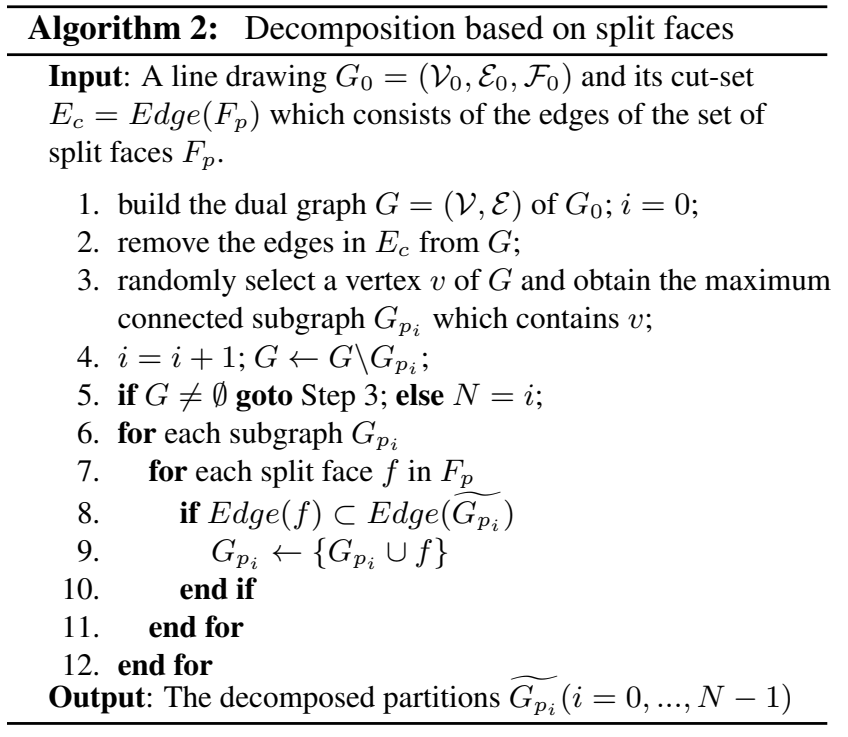

drawing based on the split faces. In the Algorithm 2, $\widetilde{G_{p_{i}}}$ and $G_{p_{i}}$ denote a common partition presented in graph $G_{0}$ and its dual graph $G$, respectively. The variable $i$ counts the decomposed partitions, and the constant $N$ saves the total number of the decomposed partitions. Steps 1-5 are used to find the decomposed partitions each of which contains several real faces of the input line drawing. Steps 6-12 are designed to merge split faces with the underlying partitions. Note that in this step, a split face $f$ is merged with the face that is coplanar and adjacent with $f$. It is obvious that the decomposition results with Algorithm 2 is unique, since the dual graph is unique and its topology is fixed.

\section{Decomposition by S-Faces}

\subsection{Criteria and Propositions for S-Faces}

Motivated by the methods proposed in the literatures on convex decomposition of a polygon [8], which decompose a polygon into simple primitives by new lines and vertices, $\mathrm{S}$-Faces in this work are also generated using new edges (i.e. external split edges and internal split edges) and new vertices (split vertices).

According to the definition of a split face, there are an infinite number of split faces on a manifold. We need to find those split faces that really simplify the reconstruction problem. The split faces are desired to separate a complex line drawing into a (approximate) minimal set of simple enough ones. In this work, the generation of a good S-Face follows these three criteria:

Criterion 1. A split edge added to form a good S-Face usually connects two collinear original edges of of the face which the split edge is on.

Criterion 2. A split edge added to form a good S-Face is usually parallel (or near parallel) to an original edge of the face which the new edge is on.

Criterion 3. A real face should have as few split edges as possible.

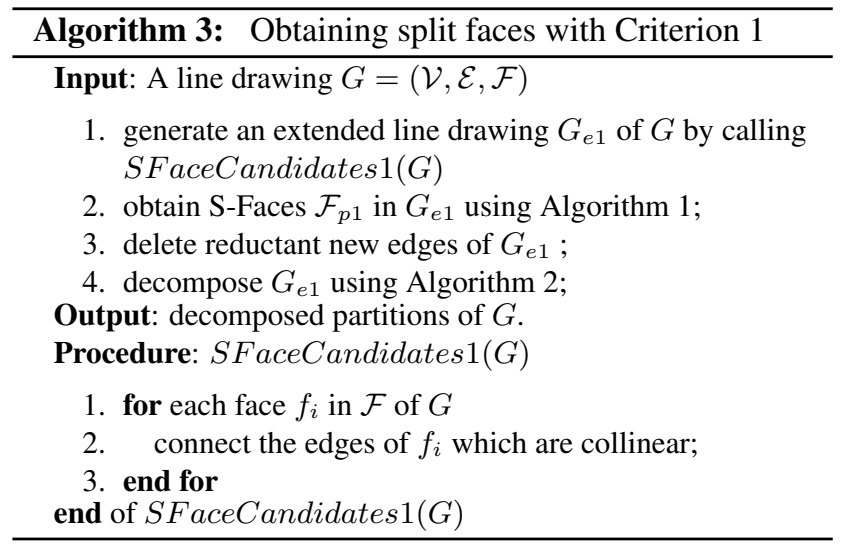

Criterion 1 and 2 are based on the fact that a large manmade object is usually formed by regular smaller objects. Criterion 3 comes from the observation that too many split edges in a real face will introduce redundant split faces, making the decomposition and reconstruction problem more complicated. Besides these criteria, we also develop several propositions to guide the generation of the $\mathrm{S}$ Faces.

Proposition 7. The degree of a split vertex passed through by one $S$-Face is 4 ;

Proof. Let $e_{12}$ be an edge shared by two neighboring faces $f_{1}$ and $f_{2}, e_{1}$ be a split edge formed by S-Face $C$ and $f_{1}$, $v^{*}$ be a split vertex formed by $e_{1} \cap e_{12}, e_{12}^{\prime}$ and $e_{12}^{\prime \prime}$ be two new edges generated by dividing $e_{12}$ with $v^{*}$, respectively, $e_{2}$ be an edge of $C$ which connects $e_{1}$ at $v^{*}$.

If $e_{2}$ is inside $f_{1}$ or coincides with one of $e_{12}^{\prime}$ and $e_{12}^{\prime \prime}$, The region enclosed by chain $\left\{e_{1}, e_{2}\right\}$ is on the surface of the manifold, which contradicts the definition of a split face. Therefore, $e_{2}$ must be inside $f_{2}$ and the degree of $v^{*}$ is 4

Proposition 8. A type 2 split edge passed through by one S-Face is coplanar with its adjacent split edges.

Proof. Let $e_{2}$ be a split edge passed through by the cycle $C$ of a S-Face, $e_{2}$ be inside a face $f_{2}, f_{1}$ and $f_{3}$ be two neighboring faces of $f_{2}$ and $f_{1} \cap f_{3}=\emptyset, f_{1}$ and $f_{3}$ respectively contain one of two endpoints of $e_{2}$. According to the proof for Proposition 7, $C$ must pass through a split edge in each of $f_{1}$ and $f_{3}$. Then, $e_{2}$ and its two adjacent split edges are passed through by $C$. Therefore, $e_{2}$ is coplanar with its adjacent split edges since the cycle $C$ of a $\mathrm{S}$-Face must be planar.

\subsection{Algorithms for S-Face-Based Decomposition}

Further decomposition of the partitions $G_{p_{i}}$, obtained by the NS-Faces as described in Section 4, includes the following two steps:

1. decomposing $G_{p_{i}}$ into simpler partitions $G_{p_{j}}^{1}$ using the $\mathrm{S}$-Faces whose new edges are generated based on Criterion 1 , 

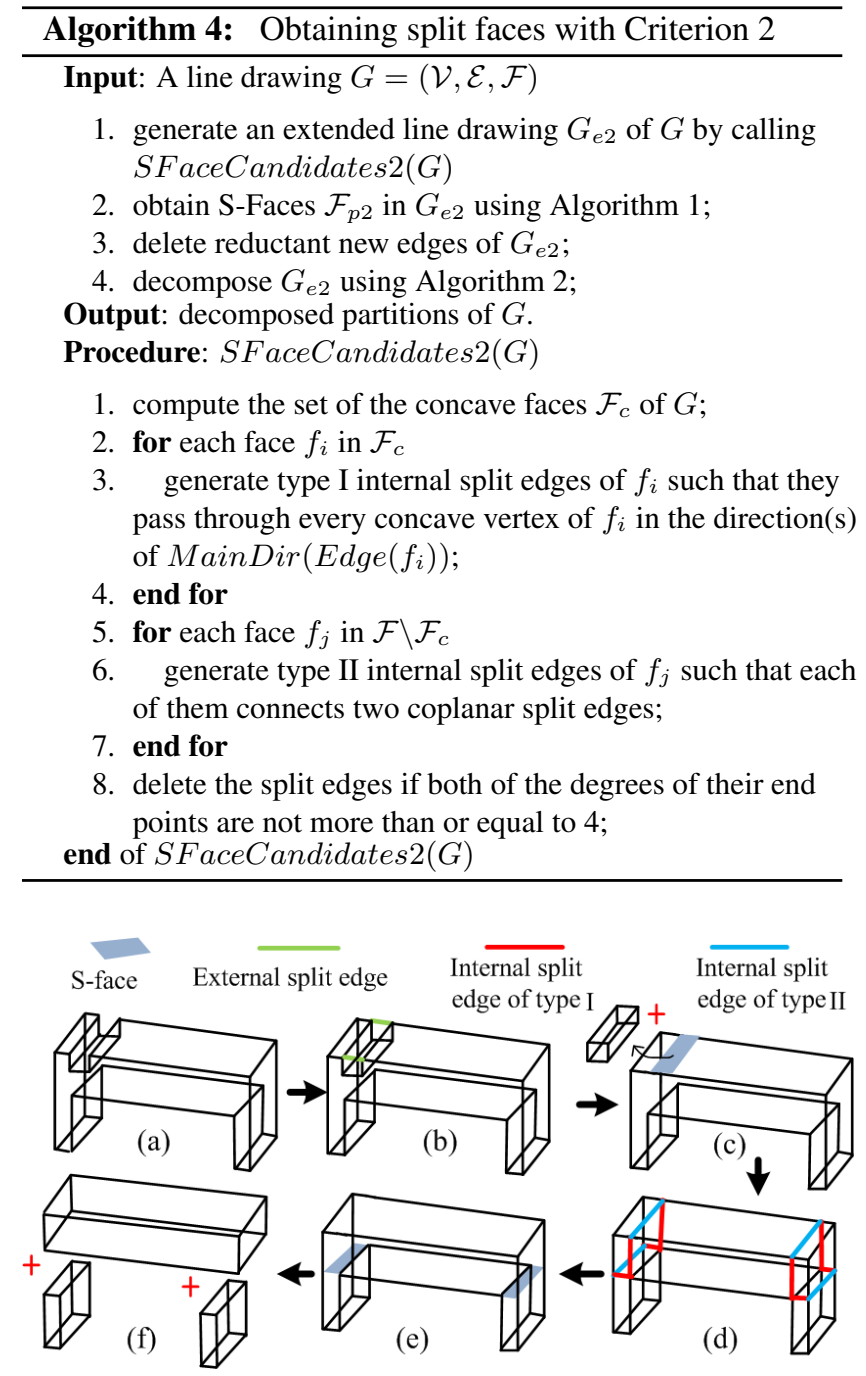

Fig. 6. S-Face-based decomposition steps. (a) A line drawing. (b) External split edges generated by Criteria 1. (c) Partitions decomposed using the S-Faces identified from the extended line drawing of Step b. (d) Internal split edges generated by Criteria 2 for the decomposed partitions of Step c. (e) The valid S-Faces are obtained with redundant new edges removed. (f) Decomposition result based on the S-Faces obtained from Step e.

2. further decomposing $G_{p_{j}}^{1}$ using the S-Faces whose new edges are generated based on Criterion 2.

These two steps are illustrated in Fig. 6 and their details are presented in Algorithm 3 and Algorithm 4, respectively. In the two algorithms, the procedures $S F$ aceCandidates 1 and $S F a c e C a n d i d a t e s 2$ are used to generate the S-Face candidates based on Criterion 1 and Criterion 2 respectively. After S-Face candidates are generated, the propositions developed for NS-Faces are also applicable for the extended line drawings in this section. Therefore, after the steps of SFaceCandidates 1 and SFaceCandidates2, we utilize Algorithm 1 to identify valid S-Faces, and employ Algorithm 2 to decompose the extended line drawing in both
Algorithm 3 and 4.

In procedure SFaceCandidates 1 , we scan all the faces and generate the new edges which connect two collinear edges in a face. The main idea of procedure SFaceCandidates2 is that: we first generate the type -I internal split edges for all the concave faces, and then generate the type-II internal split edges for the remaining faces to form S-Face candidates.

In Algorithm 4, MainDir $\left(\operatorname{Edge}\left(f_{i}\right)\right)$ denotes the direction(s) shared by the majority edges of $f_{i}$, or the direction(s) hold by the longest edge when each direction is shared by equal number of edges. In SFaceCandidates 2 , it is possible that there are multiple $\mathrm{S}$-Faces passing through one vertex, as shown in Fig 6d. According to Criterion 3, we only preserve one of these S-Faces (in practical implementation, only the S-Face with a minimum number of new edges or a minimum sum of the length of new edges of these S-Faces is preserved for the decomposition step). In both $S F a c e C a n d i d a t e s 1$ and SFaceCandidates 2 , the new edges of the removed S-Faces and those ones outside identified split faces are deleted before the decomposition step. Note that Proposition 8 is used in Step 8 of SFaceCandidates 2 to avoid the generation of the S-Face candidates which cannot be S-Faces, Proposition 7 is used to delete the new edges which cannot form complete cycles of S-Face candidates.

\section{3D Reconstruction from a Line Drawing}

After separating a line drawing using its split faces, a set of simpler line drawings is obtained. It is not difficult to deal with $3 \mathrm{D}$ reconstruction from these separated simple line drawings. Several framework, such as [11], [20], can be used to further reconstruct a complete 3D object from these simpler line drawings. In this work, we use the examplebased reconstruction method [20] to carry out the reconstruction, since it is robust to sketch errors and can provide a parametric 3D object. We reconstruct a 3D object from these simpler line drawings by these steps:

1. selecting multiple 3D model candidates from the model database for each simpler line drawing,

2. deriving a complete 3D object using a maximum-aposteriori estimation that selects the best $3 \mathrm{D}$ model candidates so that the reconstructed result fits the input line drawing best.

Our experimental results show that the proposed decomposition algorithm can well cooperate with the example-based reconstruction method, since a complex line drawing can usually be separated into simple enough ones, only a limited number of examples in the 3D model database can handle the reconstructions from most of the line drawings which represent planar manmade manifolds. 

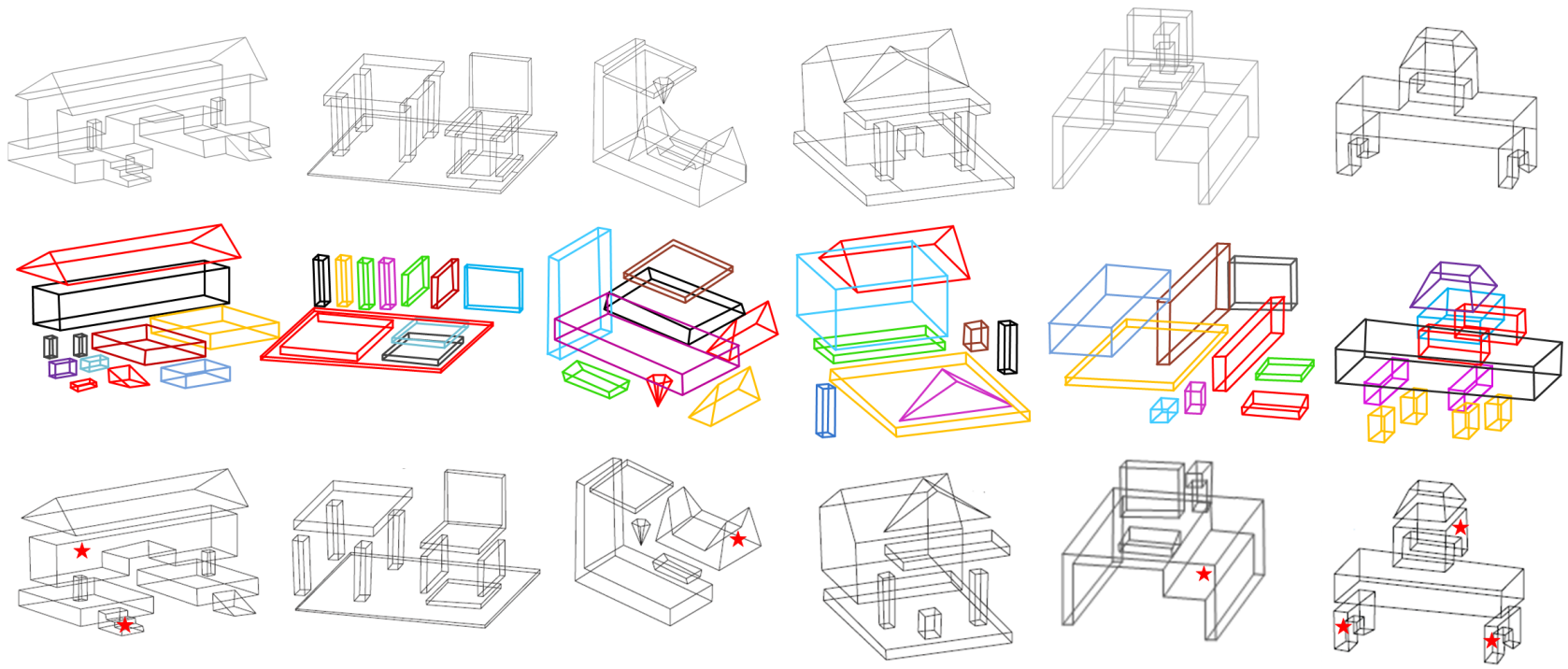

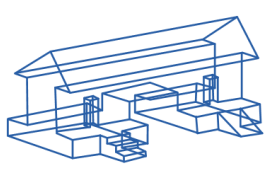

(a)

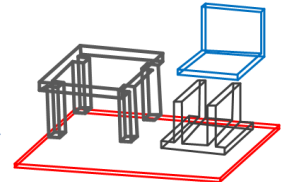

(b)

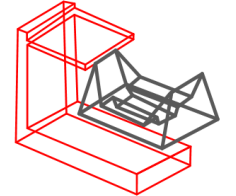

(c)

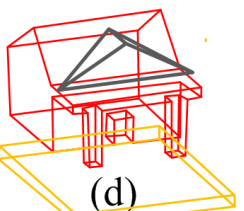

(d)

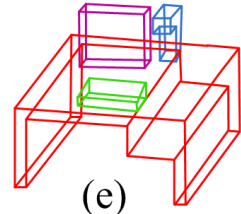

(e)

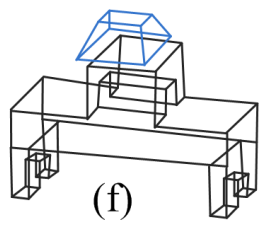

Fig. 7. Decomposition results on line drawings $(a)-(f)$. The second, third, and fourth columns show the separation results from SFM, OCM, and IFM, respectively.

Tabel 1. Numbers of the separated partitions of the examples in Fig. 7 by SFM, OCM, and IFM.

\begin{tabular}{|c|c|c|c|c|c|c|}
\hline Algorithm & (a) & (b) & (c) & (d) & (e) & (f) \\
\hline SFM & 11 & 11 & 8 & 8 & 9 & 11 \\
\hline OCM & 8 & 10 & 5 & 7 & 4 & 5 \\
\hline IFM & 1 & 4 & 2 & 3 & 4 & 2 \\
\hline
\end{tabular}

\section{Experiments}

In this section, we conduct experiments to evaluate the effectiveness and efficiency of our split face based line drawing separation method (SFM). The internal face based separation method in [11] (IFM) and the object cut based one in [21] (OCM) are used as the baselines. The proposed method is implemented in $\mathrm{C}++$ on a standard $2.53 \mathrm{GHz}$ i5 CPU machine and only one core is used.

In the experiment, we conduct the proposed method on dozens of line drawings collected from [21] and other related literatures. Some example line drawings and their separation results from the three separation methods (SFM, OCM and IFM) are shown in Fig. 7 and Table 1. As can be seen from the second column of Fig. 7 and Table 1, our algorithm successfully separate line drawings into much simpler partitions than IFM. Compared to the decomposed results of OCM (the third column of Fig. 7), all the results of SFM correspond to simple partitions that represent primitive 3D shape parts, such as cuboid and prisms while some com- plex partitions are still shown in the results of OCM (e.g., the partitions marked by red stars).

In Fig. 8, four other general line drawings and the numbers of the separation results are shown, together with the reconstruction results from SFM. The results indicate that SFM can separate more general line drawings, compared to IFM and OCM (e.g, the line drawing in Fig. 8(c) can be separated into 20 partitions by SFM, while that number by IFM and OCM are 1 and 3, respectively). Therefore, we can draw the conclusion that the combination of SFM and the example-based reconstruction algorithm is able to robustly reconstruct wider range of parametric solid objects than the method in [20] (the parametric 3D shape cannot be achieved if its corresponding partition has no match in the example database). The computational time of the proposed separation algorithm depends on the complexity of a line drawing. Each test line drawing in Fig. 7 is decomposed within 1 second, which is much more efficient than both IFM and OCM.

\section{Conclusions}

In this paper, we propose a line drawing separation method which uses split faces to decompose a line drawing into simpler ones. A split face in this work is obtained by both the topological information in an original line drawing and the derived new topological information. Therefore, it can be employed to obtain a better separation 
(a)
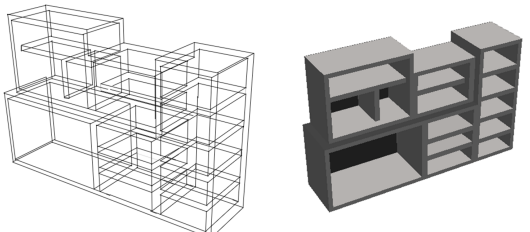

$\left[N_{I F M}, N_{S F M}, N_{O C M}\right]=[2,1226]$
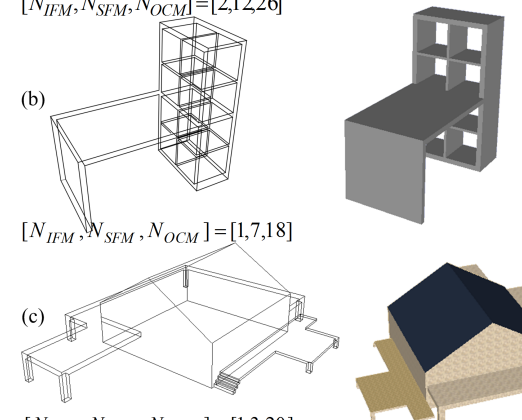

$\left[N_{I F M}, N_{S F M}, N_{O C M}\right]=[1,3,20]$
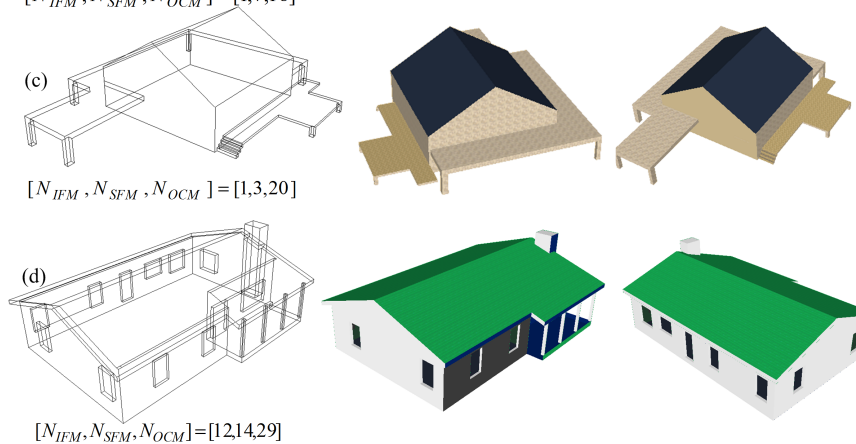

Fig. 8. Four other example line drawings (note that the artificial lines in the line drawings are hidden for concision), and the reconstructed 3D objects, each shown from two views. The number of the decomposed partitions based on IFM, OCM, and SFM are respectively denoted by $N_{I F M}, N_{O C M}$, and $N_{S F M}$.

of a complex line drawing than previous related methods. The proposed separation method is important for the 3D reconstruction from a complex line drawing, since the task of recovering a complete 3D object will be very easy when a complex line drawing is decomposed into simple ones. The proposed line drawing decomposition method can also be applied in other related applications, such as model-based (sketch-based) 3D model retrieval. We also develop efficient algorithms for identification of a split face and decomposition based on split faces. The experiments show that the proposed separation method combined with the example-based reconstruction algorithm can robustly reconstruct more complex parametric solid objects than previous methods.

\section{Acknowledgement.}

This work was supported by grants from Science, Industry, Trade, and Information Technology Commission of Shenzhen Municipality (No. JC201005270378A), Guangdong Innovative Research Team Program (No. 201001D0104648280), Shenzhen Basic Research Program (JCYJ20120617114614438, JC201005270350A, JCYJ20120903092050890), Scientific Research Fund of Hunan Provincial Education Department (No. 13C073),
Industrial Technology Research and Development Program of Hengyang Science and Technology Bureau (No. 2013KG75), and the Construct Program of the Key Discipline in Hunan Province. The authors are thankful to Huixuan Tang for her valuable suggestions.

\section{References}

[1] M. Armstrong. Basic Topology. Springer, 1983.

[2] S.C. Agarwal and J.W.N. Waggenspack. Decomposition Method for Extracting Face Topologies from Wireframe Models, Computer- Aided Design, 24(3):123-140, 1922.

[3] S. Bagali and J. Waggenspack. A shortest path approach to wireframe to solid model conversion. Proc. 3rd Symp. Solid Modeling and Applications, pp. 339-349, 1995.

[4] P. Company, A. Piquer, et al. A survey on geometrical reconstruction as a core technology to sketch-based modeling. Computers\&Graphics, 29(6):892-904, 2005.

[5] P. Company, M. Contero, J. Conesa, and A. Piquer. An OptimisationBased Reconstruction Engine for 3D Modeling by Sketching. Computers \& Graphics, vol. 28, pp. 955-979, 2004.

[6] M.R. Gary and D.S. David. Computers and Intractability: A Guide to the Theory of NP-completeness. W. H. Freeman, 1979.

[7] I. Grimstead and R. Martin. Creating Solid Models from Single 2D Sketches. Proc. 3th ACM symposium on Solid modeling and applications, pp. 323-337, 1995.

[8] J.M. Keil. Polygon decomposition. Handbook of Computational Geometry, vol. 2, pp. 491-518, 2000.

[9] H. Lipson and M. Shpitalni. Optimization-based reconstruction of a 3D object from a single freehand line drawing. Computer-Aided Design, 28(8):651-663, 1996.

[10] Y. Leclerc and M. Fischler. An Optimization-Based Approach to the Interpretation of Single Line Drawings as 3D Wire Frames. Int'l Journal of Computer Vision, 9(2):113-136, 1992.

[11] J. Liu, Y. Chen, and X. Tang. Decomposition of Complex Line Drawings with Hidden Lines for 3D Planar-Faced Manifold Object Reconstruction. PAMI, 33(1):3-15, 2011.

[12] J. Liu, L. Cao, Z. Li, and X. Tang. Plane-Based Optimization for 3D Object Reconstruction from Single Line Drawings. PAMI, 30(2):315-327, 2008.

[13] J. Liu, Y. Lee, and W.-K. Cham. Identifying Faces in a 2D Line Drawing Representing a Manifold Object. PAMI, 24(12):1579-1593, 2002.

[14] P. Min, J. Chen, and T. Funkhouser. A 2Dsketch interface for a 3D model search engine. SIGGRAPH, Technical Sketches, 2002.

[15] T. Marill. Emulating the Human Interpretation of Line Drawings as Three-Dimensional Objects. Int'l Journal of Computer Vision, 6(2):147-161, 1991.

[16] S. Ortiz. 3D searching starts to take shape. Computer, 37(8): 24-26, 2004.

[17] L. Ros and F. Thomas. Overcoming Superstrictness in Line Drawing Interpretation. PAMI, 24(4):456-466, 2002.

[18] A. Shesh and B. Chen. Smartpaper: An interactive and user friendly sketching system. Computer Graphics Forum, 23(3):301-310, 2004.

[19] A. Turner, D. Chapman, and A. Penn. Sketching Space. Computers \& Graphics, 24(6):869-879, 2000.

[20] T. Xue, J. Liu, and X. Tang. Example-based 3D object reconstruction from line drawings. IEEE Proc. CVPR, 2012.

[21] T. Xue, J. Liu, and X. Tang. Object cut: Complex 3D object reconstruction through line drawing separation. IEEE Proc. CVPR, 2010.

[22] C. Zou, J. Liu, and J. Liu. Precise 3D Reconstruction from a Single Image. $A C C V, 2012$.

[23] C. Zou and J. Liu. The method for line drawing separation. Technicle Report, the Media Lab, Shenzhen Institutes of Advanced Technology, 2014. 